\title{
Ventilation-perfusion inequality in the human lung is not increased following no-decompression-stop hyperbaric exposure
}

\author{
Gaea Schwaebe Moore $\cdot$ Stewart C. Wong • \\ Chantal Darquenne $\cdot$ Tom S. Neuman · John B. West • \\ G. Kim Prisk
}

Accepted: 27 July 2009 / Published online: 19 August 2009

(C) The Author(s) 2009. This article is published with open access at Springerlink.com

\begin{abstract}
Venous gas bubbles occur in recreational SCUBA divers in the absence of decompression sickness, forming venous gas emboli (VGE) which are trapped within pulmonary circulation and cleared by the lung without overt pathology. We hypothesized that asymptomatic VGE would transiently increase ventilation-perfusion mismatch due to their occlusive effects within the pulmonary circulation. Two sets of healthy volunteers $(n=11, n=12)$ were recruited to test this hypothesis with a single recreational ocean dive or a baro-equivalent dry hyperbaric dive. Pulmonary studies (intrabreath $V_{\mathrm{A}} / Q(\mathrm{iV} / Q)$ ), alveolar dead space, and FVC) were conducted at baseline and repeat 1- and 24-h after the exposure. Contrary to our hypothesis $V_{\mathrm{A}} / Q$ mismatch was decreased 1-h post-SCUBA dive (iV/Q slope $0.023 \pm 0.008 \mathrm{ml}^{-1}$ at baseline vs. $0.010 \pm 0.005$ NS), and was significantly reduced 24 -h post-SCUBA dive $(0.000 \pm 0.005, p<0.05)$, with improved $V_{\mathrm{A}} / Q$ homogeneity inversely correlated to dive severity. No changes in $V_{\mathrm{A}} / Q$ mismatch were observed after the chamber dive. Alveolar dead space decreased 24-h post-SCUBA dive (78 $\pm 10 \mathrm{ml}$ at baseline vs. $56 \pm 5, p<0.05$ ), but not 1 -h post dive. FVC rose 1 -h post-SCUBA dive $(5.01 \pm 0.181$ vs. $5.21 \pm 0.26, p<0.05)$, remained elevated 24 -h post
\end{abstract}

G. S. Moore · S. C. Wong · C. Darquenne · J. B. West ·

G. Kim Prisk

Department of Medicine, University of California,

San Diego, USA

T. S. Neuman

Department of Emergency Medicine,

University of California, San Diego, USA

G. S. Moore $(\square)$

4860 Y Street, Suite 2500, Sacramento, CA 95817, USA

e-mail: gaeamoore@gmail.com
SCUBA dive $(5.06 \pm 0.2, p<0.05)$, but was decreased 1 -hr after the chamber dive $(4.96 \pm 0.31 \mathrm{~L}$ to $4.87 \pm 0.32$, $p<0.05)$. The degree of $V_{\mathrm{A}} / Q$ mismatch in the lung was decreased following recreational ocean dives, and was unchanged following an equivalent air chamber dive, arguing against an impact of VGE on the pulmonary circulation.

Keywords $V_{\mathrm{A}} / Q \cdot \mathrm{SCUBA} \cdot$ Venous gas emboli $\cdot$ VGE . Decompression sickness · Pulmonary function

\section{Introduction}

Decompression sickness is a well-described risk of SCUBA diving in which nitrogen gas dissolved within tissue and blood evolves into gas bubbles upon ascent, resulting in tissue injury (Marx 2006). While decompression sickness has become relatively rare with improved diver training and adherence to published dive tables, small asymptomatic venous gas emboli (VGE) are common in the ascending recreational diver (Dujic et al. 1993; Jankowski et al. 1997; Carturan et al. 2002; Dunford et al. 2002; Valic et al. 2004; Dujic et al. 2005c). The gas contents of VGE are released from pulmonary circulation into neighboring alveoli and eliminated though exhalation (Thorsen et al. 1995). While the presence of venous gas microemboli has been associated with decreased diffusing capacity of the lung for carbon monoxide $\left(D_{\mathrm{L}} \mathrm{CO}\right)$ and $\mathrm{PaO}_{2}$ (Dujic et al. 1993), no reported human studies have demonstrated whether transient occlusion of pulmonary capillaries during clearance of VGE alters the distribution of ventilation perfusion ratios $\left(V_{\mathrm{A}} / Q\right)$ in the healthy human lung following recreational no-decompression stop ocean SCUBA diving. We hypothesized that following a single non-decompression stop ocean SCUBA dive, gas exchange would be sufficiently affected 
by transient venous gas micro-emboli within pulmonary vasculature that a measurable increase in ventilationperfusion inequality would be found, followed by rapid resolution.

\section{Methods}

\section{Subjects}

A total of 23 healthy volunteers were recruited for this study at two separate time points. Eleven certified recreational SCUBA divers were first enrolled in the openwater study, followed by 12 college students and staff for the dry hyperbaric chamber experiment. The participants in the SCUBA study group (8 males, 3 females) were $31 \pm 5$ years old, with height $175 \pm 6 \mathrm{~cm}$, weight $75 \pm 14 \mathrm{~kg}$, and $\mathrm{FEV}_{1}(\%$ predicted) $104 \pm 14$. The participants in the hyperbaric study group (6 male, 6 female) were $30 \pm 7$ years old, with height $178 \pm 9 \mathrm{~cm}$, weight $80 \pm 19 \mathrm{~kg}$, and $\mathrm{FEV}_{1}$ (\% predicted) $106 \pm 14$. Exclusion criteria included a history of smoking, lung disease, and any condition that might preclude diving or hyperbaric exposure. No subjects withdrew from the study before completion or were excluded from analysis, and none developed decompression sickness or associated symptoms. The study was reviewed by the UCSD Human Research Protection Program, and written consent was obtained prior to participation.

\section{Scuba dive study design}

11 healthy volunteers performed a single recreational non-saturation SCUBA ocean dive in the ocean waters off San Diego, CA between June and August 2002. Ocean temperatures were $17.5-23.0^{\circ} \mathrm{C}$ at the surface and $13.3-$ $23.0^{\circ} \mathrm{C}$ at a depth of $5.5 \mathrm{~m}$ (UCSD 2007). Divers wore a 7-mm wet suit, a SCUBA apparatus, and a dive computer (Suunto models Viper and Mosquito, Vantaa, Finland). The divers chose their own time-depth profiles but were instructed to maximize their dive intensity to the threshold of a decompression-stop. Exercise was not controlled for, including exercise prior to, during or after the dive. Baseline pulmonary function data were collected within 4 days prior to this dive, and then repeated approximately one hour and 24 hours after surfacing. Subjects did not perform other dives between pre-dive testing and our post-dive measurement.

The dive profile was downloaded from the Suunto dive computers to a PC for analysis and calculation of dive severity, which was determined using the model outlined by Short and Flahan (1989) based on the US Navy Dive Table (1955). In this model, dive severity is calculated in six theoretic tissue compartments (defined by half-times of nitrogen saturation: $5,10,20,40,80$, or $120 \mathrm{~min}$ ). A fractional saturation (or "dive severity") of 1.0 in any of the six "compartments" would imply that the quantity of nitrogen was high enough to require staged decompression. The unique time-depth dive profiles were plotted minute-by-minute in each "tissue compartment." The maximum fractional saturation of nitrogen calculated in any compartment during a dive was taken as that dive's severity.

Hyperbaric chamber study design

Twelve healthy volunteers from the second wave of recruitment underwent a single dry non-decompression stop hyperbaric chamber dive at the University of California, Sand Diego Hillcrest Hospital, which houses a multi-place hyperbaric chamber $7.0 \mathrm{~m}$ (23 in.) long and $1.95 \mathrm{~m}$ (6 in.) in diameter. A fixed protocol was conducted with compression to $18.3 \mathrm{~m}$ sea water ( $60 \mathrm{ft}, 2.77$ ATA) within $5 \mathrm{~min}$, with maintenance at that pressure for the remainder of 60 min before decompression to atmospheric pressure in 4 min. Baseline pulmonary function data were collected using the same techniques as the open-water study, except that baseline measurements were collected within $24 \mathrm{~h}$ of the scheduled hyperbaric chamber session. Volunteers remained dry and were accompanied in the chamber by a trained hyperbaric chamber technician. Exertion was controlled for by having the divers remain seated during the hyperbaric exposure, by mandating slow-walking through the hospital to the lobby, and by providing door-to-door transportation from the hospital to the laboratory facility where pulmonary measurements were conducted. In addition, volunteers were asked not to exercise between the initial hyperbaric exposure and the 24-h post-dive data-point. Collection of pulmonary function data and calculation of dive severity were conducted in the same manner as in the SCUBA portion of the study.

To determine whether the compression/decompression component of the dry hyperbaric experience would alter pulmonary function, a control group (made up of half of the hyperbaric study volunteers, $n=6$ ) performed a minimalintensity dry-hyperbaric dive 1 month later. These volunteers were again pressurized in the hyperbaric chamber to $18.3 \mathrm{~m}$ sea water (60 ft, 2.77 ATA) within $5 \mathrm{~min}$, then

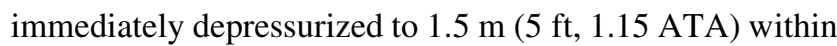
$4 \mathrm{~min}$ where they spent the remainder of $60 \mathrm{~min}$ before decompression to atmospheric pressure. This dive profile (subsequently referred to as 60-5) carried virtually no nitrogen loading, and thus a minimal chance for VGE formation. Collection and analysis of data, as well as exercise restrictions were identical to the protocol described for the 60-60 hyperbaric chamber dive as above. 
Pulmonary function testing

Tests included the range of $V_{\mathrm{A}} / Q(\mathrm{iV} / Q)$ and spirometry. $D_{\mathrm{L}} \mathrm{CO}$ was also assessed for the hyperbaric chamber group. Ventilation/perfusion inequality distribution in the lung $\left(V_{\mathrm{A}} / Q\right)$ was determined with data collected from a bag-inbox apparatus (Prisk et al. 1995). The subjects breathed air through a mouthpiece for 60-120 s while flows and gas concentrations were recorded at the lips at $100 \mathrm{~Hz}$. During this period, the subjects' oxygen consumption $\left(\mathrm{VO}_{2}\right)$ and carbon dioxide production $\left(\mathrm{VCO}_{2}\right)$ were determined. Gas flow rates were measured using a Fleisch No. 2 pneumotachograph in the wall of the box coupled to a differential pressure transducer (Validyne MP-45, Northridge, California). Gas concentrations were measured with a respiratory mass spectrometer (Marquette MGA-1100, Milwaukee, WI). The alveolar dead space (physiological minus anatomical) was also derived from these data and averaged over all breaths (Prisk et al. 1995).

The subjects then rapidly inhaled to TLC, and then exhaled at a controlled rate of $0.5 \mathrm{l} / \mathrm{s}$ to RV. From expired gas concentrations during this slow vital capacity expiration, the instantaneous respiratory exchange ratio was calculated throughout the exhalation which was transformed into an intra-breath $V_{\mathrm{A}} / Q$ curve $(\mathrm{iV} / Q)$, from which the range of distribution of ventilation-perfusion ratios in the lung was inferred (Guy et al. 1976; Prisk et al. 2003). During our analysis of $\mathrm{iV} / Q$, we considered the slope of Phase III of the $\mathrm{iV} / Q$ curve as a measure of $V_{\mathrm{A}} / Q$ inequality as this has previously been shown to correlate closely with independent measurements of $V_{\mathrm{A}} / Q$ inequality in the lung (Prisk et al. 2003).

Forced spirometry was performed using Spirocard ${ }^{\circledR}$ on a laptop computer (QRS Diagnostic, Plymouth, MN, USA). Volumes were corrected to BTPS. All subjects performed the tests at the scheduled time points in triplicate, and the highest value for each triplet was chosen.

The diffusing capacity of the lung for carbon monoxide $\left(D_{\mathrm{L}} \mathrm{CO}\right)$ was added to our pulmonary function protocol after the SCUBA portion of our study was completed; as such data were collected for the hyperbaric subjects only. Rebreathing measurements were performed according to the method of Sackner et al. (1975) with rebreathing lasting for 15 seconds at approximately 30 breaths per minute. Inspired gas contained $0.3 \% \mathrm{C}^{18} \mathrm{O}, 10 \%$ argon, and $21 \%$ $\mathrm{O}_{2}, 68.7 \% \mathrm{~N}_{2}$ with calibrations performed daily.

\section{Statistical analysis}

Data were grouped by dive type, subject, and dive status (pre-dive, 1-h post-dive, 24-h post-dive). Statistical analysis was performed using Systat V5.0 (Systat, Evanston, IL, USA). Two-way ANOVA was performed to test for differences between subjects and type of dive. Tests with signifi- cant $\mathrm{F}$ ratios were analyzed for significance using post hoc pair wise comparisons using the Bonferroni adjustment. Significance was accepted at $p<0.05$.

\section{Results}

Dive severity

The average time-depth profile for the 11 recreational divers was $48 \mathrm{~min}$ with a maximum depth of $30 \mathrm{~m}$ (98 fsw). These profiles varied with an underwater time of 30-70 min, maximum depth from 19-44 m, and dive severity index ranging from $0.45-0.92$. The average dive severity index of the ocean SCUBA divers was 0.69. In the hyperbaric study, dive severity index for the $60 \mathrm{~min}-60 \mathrm{ft}$ dive was 0.94 . For the $60 \mathrm{~min}-5 \mathrm{ft}$ control study, the dive severity index was 0.12 .

\section{Pulmonary function}

The pulmonary function data are shown in Table 1.

\section{$V_{A} / Q$ mismatch}

In the SCUBA group, the degree of $V_{\mathrm{A}} / Q$ mismatch (measured as the slope of phase III along the $\mathrm{iV} / Q$ curve) decreased significantly the day following the dive $(0.023 \pm 0.008$ baseline vs. $0.000 \pm 0.005, p<0.05)$, but not immediately after the dive (see Table 1; Fig. 1). The net decrease in $V_{\mathrm{A}} / Q$ mismatch $(\mathrm{iV} / Q)$ from baseline to 24 -h was inversely correlated with the dive severity index, with the more severe dives associated with less improvement in $V_{\mathrm{A}} / Q$ mismatch (see Fig. 2). The hyperbaric chamber $60 \mathrm{~min}-60 \mathrm{ft}$ dive group data, which had the greatest dive severity (0.94), demonstrated no significant changes between baseline and post-dive measurements of $\mathrm{iV} / Q$.

\section{Alveolar dead space}

In parallel with the changes in $\mathrm{iV} / Q$, there was a significant decrease in alveolar dead space 24-h following the SCUBA dive, but not at the 1 -h post-dive time point (see Table 1). Alveolar dead space decreased by $22 \mathrm{ml}$ from baseline to the 24 -h post-dive read $(78 \pm 10$ vs. $56 \pm 5 \mathrm{ml}, p>0.05)$ and by $31 \mathrm{ml}$ from the 1 - to 24 -h time points $(87 \pm 12 \mathrm{ml}$ vs. $56 \pm 5, p<0.05)$. There were no significant changes in alveolar dead space $1 \mathrm{~h}$ after the SCUBA dive, or at any time point following the hyperbaric exposure.

\section{FVC}

The average FVC of divers in the SCUBA arm rose significantly from the pre-dive to 1-h time point with an increase 
Table 1 Pulmonary function following SCUBA and hyperbaric exposure
Data are mean \pm SE. Data were collected in triplicate at pre-dive, 1- and 24-h post-dive time points. Hyperbaric (60-5) and hyperbaric (60-60) compare same $n=6$ subjects under different conditions.

* Significantly different from pre-dive time point $p<0.05$

$\dagger$ Significantly different from 1 -h post-dive time point $p<0.05$

\begin{tabular}{|c|c|c|c|}
\hline & Pre-Dive & 1-h post & $24 \mathrm{~h}$ post \\
\hline \multicolumn{4}{|l|}{$\mathrm{iV} / Q$ slope } \\
\hline \multicolumn{4}{|l|}{ Phase III (ml-1) } \\
\hline SCUBA, $n=11$ & $0.023 \pm 0.008$ & $0.010 \pm 0.005$ & $0.000 \pm 0.005^{* \dagger}$ \\
\hline HYPERBARIC $60-60, n=12$ & $0.066 \pm 0.016$ & $0.065 \pm 0.007$ & $0.077 \pm 0.013$ \\
\hline HYPERBARIC $60-5, n=6$ & $0.113 \pm 0.046$ & $0.073 \pm 0.013$ & $0.147 \pm 0.057$ \\
\hline \multicolumn{4}{|l|}{ Alveolar dead space (ml) } \\
\hline SCUBA, $n=10$ & $78 \pm 10$ & $87 \pm 12$ & $56 \pm 5^{* \dagger}$ \\
\hline HYPERBARIC $60-60, n=6$ & $0.087 \pm 0.032$ & $0.060 \pm 0.014$ & $0.070 \pm 0.010$ \\
\hline HYPERBARIC $60-60, n=12$ & $36 \pm 2$ & $35 \pm 2$ & $37 \pm 2$ \\
\hline HYPERBARIC $60-5, n=6$ & $33 \pm 2$ & $38 \pm 2.49$ & $36 \pm 2$ \\
\hline HYPERBARIC 60-60, $n=6$ & $40 \pm 2$ & $36 \pm 2$ & $39 \pm 1$ \\
\hline \multicolumn{4}{|l|}{ Spirometry } \\
\hline \multicolumn{4}{|l|}{$\mathrm{FVC}(\mathrm{L})$} \\
\hline SCUBA, $n=11$ & $5.01 \pm 0.18$ & $5.21 \pm 0.26^{*}$ & $5.06 \pm 0.20^{* \dagger}$ \\
\hline HYPERBARIC $60-60, n=12$ & $4.96 \pm 0.31$ & $4.87 \pm 0.32 *$ & $4.84 \pm 0.32$ \\
\hline HYPERBARIC $60-5, n=6$ & $5.29 \pm 0.49$ & $5.32 \pm 0.50$ & $5.27 \pm 0.44$ \\
\hline HYPERBARIC $60-60, n=6$ & $5.40 \pm 0.40$ & $5.34 \pm 0.40$ & $5.31 \pm 0.46$ \\
\hline \multicolumn{4}{|l|}{$\mathrm{FEV}_{1}(\mathrm{~L})$} \\
\hline SCUBA, $n=11$ & $4.01 \pm 0.13$ & $4.16 \pm 0.19^{*}$ & $4.04 \pm 0.14 * \dagger$ \\
\hline HYPERBARIC $60-60, n=12$ & $4.04 \pm 0.22$ & $3.91 \pm 0.20$ & $3.91 \pm 0.21$ \\
\hline HYPERBARIC $60-5, n=6$ & $4.19 \pm 0.27$ & $4.18 \pm 0.027$ & $4.18 \pm 0.29$ \\
\hline HYPERBARIC $60-60, n=6$ & $4.30 \pm 0.26$ & $4.20 \pm 0.20$ & $4.22 \pm 0.25$ \\
\hline \multicolumn{4}{|l|}{ FEF $25-75 \%(1 / s)$} \\
\hline SCUBA, $n=11$ & $4.13 \pm 0.22$ & $4.19 \pm 0.24 *$ & $4.15 \pm 0.25$ \\
\hline HYPERBARIC $60-60, n=12$ & $4.21 \pm 0.33$ & $4.13 \pm 0.3$ & $3.99 \pm 0.31$ \\
\hline HYPERBARIC $60-5, n=6$ & $4.10 \pm 0.34$ & $4.04 \pm 0.29$ & $4.11 \pm 0.46$ \\
\hline HYPERBARIC $60-60, n=6$ & $4.35 \pm 0.44$ & $4.30 \pm 0.42$ & $4.1 \pm 0.35$ \\
\hline \multicolumn{4}{|l|}{$D_{\mathrm{L}} \mathrm{CO}$} \\
\hline SCUBA, $n=11$ & NA & NA & NA \\
\hline HYPERBARIC $60-60, n=12$ & $23.5 \pm 1.3$ & $23.6 \pm 1.1$ & $23.9 \pm 1.1$ \\
\hline HYPERBARIC $60-5, n=6$ & $26.3 \pm 1.1$ & $26.9 \pm 1.1$ & $26.3 \pm 1.1$ \\
\hline HYPERBARIC $60-60, n=6$ & $23.9 \pm 0.7$ & $24.8 \pm 0.8$ & $24.4 \pm 0.7$ \\
\hline
\end{tabular}

of $4 \%$ or $0.21(5.01 \pm 0.18$ vs. $5.21 \pm 0.261, p<0.05)$ (Table 1). While FVC returned towards baseline by the 24-h mark, it remained significantly elevated at this time point $(5.01 \pm 0.181$ vs. $5.06 \pm 0.2, p<0.05)$. There were parallel changes in both $\mathrm{FEV}_{1}$ and $\mathrm{FEF}_{25-75 \%}$ at the 1- and 24-h marks. In the hyperbaric study, the only change seen was a small reduction in FVC from $4.96 \pm 0.31$ to $4.87 \pm 0.321$ 1 -h after exposure $(p<0.05)$; however, there were no significant changes in other spirometry indices.

\section{$D_{L} C O$}

The diffusing capacity of the lung for carbon monoxide was measured only in the hyperbaric study. No significant changes were observed between any time points in the hyperbaric dive group (Table 1).
Hyperbaric control

In the hyperbaric control study, there were no significant changes in any measures of pulmonary function observed between the subset of six subjects who performed both the $60 \mathrm{~min}-60 \mathrm{ft}$ dives and the $60 \mathrm{~min}-5 \mathrm{ft}$ dives. The results for this subset of the hyperbaric group are presented separately in Table 1.

\section{Discussion}

This two-part study was designed to assess changes in $V_{\mathrm{A}} / Q$ mismatch following a single ocean SCUBA dive or dry hyperbaric exposure. While an increase in $V_{\mathrm{A}} / Q$ mismatch was hypothesized, we found that $V_{\mathrm{A}} / Q$ mismatch was 


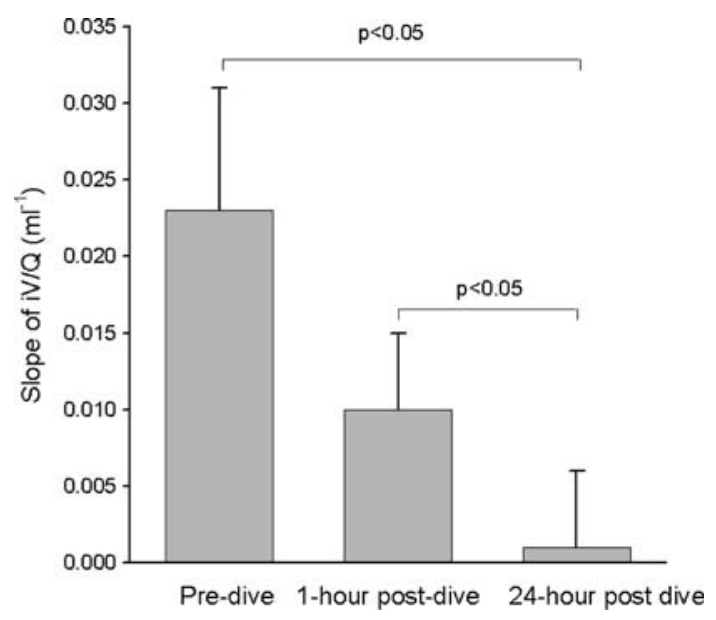

Fig. 1 Average $i V / Q$ Phase III slope for the SCUBA group at the predive, 1 and 24 -h post-dive time points. A significant decrease in the slope was seen between the pre-dive and 24 -h post-dive time point, indicating a decrease in ventilation perfusion mismatch $(p<0.05)$. Error bars are SE

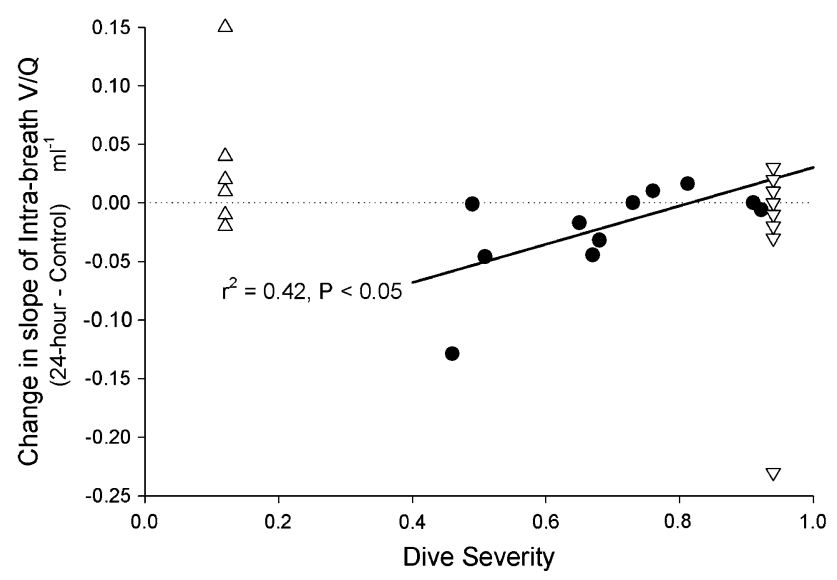

Fig. 2 Correlation between dive severity and change in $\mathrm{iV} / Q$ between the pre-dive and 24-h post-dive time points. As dive severity increased, the change in $\mathrm{iV} / Q$ Phase III slope seen between the two time points decreased. Overall, there was a significant positive correlation between these two sets of data $\left(r^{2} 0.42, p<0.05\right)$. Filled circle SCUBA dive. Downward triangle Hyperbaric 60-60 group. Upward triangle Hyperbaric 60-5 group

reduced after ocean diving without change following hyperbaric exposure, suggesting that any changes in $V_{\mathrm{A}} / Q$ are multifactorial and VGE (if indeed they were present in this study) do not significantly alter ventilation-perfusion mismatch following ascent.

\section{Study design}

VGE form after ascent from diving and are cleared by the lung (Thorsen et al. 1995). We predicted post-dive VGEassociated disruption in capillary circulation sufficient to increase $V_{\mathrm{A}} / Q$ mismatch. When the distribution of $V_{\mathrm{A}} / Q$ inhomogeneity instead improved after SCUBA diving, a follow-up hyperbaric study was designed to isolate the effects of nitrogen load and decompression (most associated with VGE formation) in order to minimize confounding factors involved with SCUBA diving. To maximize the possibility of venous gas bubble formation while not endangering our volunteers, the hyperbaric dive profile was designed to reach but not exceed the no-decompressionstop limits of accepted dive tables. The hyperbaric exposure subsequently referred to as the 60-60 dive profile was at the approximate limit of U.S. Navy guidelines for a no-decompression stop dive (Direction of Commander, N. S. S. C. 2008) as evidenced by a dive severity index of 0.94 ; in the model we utilized (Short 1989), a fractional saturation of nitrogen, or "dive severity," of 1.0 in any compartment would require staged-decompression. Similar work has reported dive severity in relation to the probability of developing decompression sickness (pDCS); however, due to the relatively low risk of decompression sickness in the dive profiles found in this study (which involved only nodecompression stop dive profiles), the pDCS would not have provided a meaningful tool for describing dive intensity. As a reference for comparison to other studies, the pDCS of the 60-60 chamber dive (the most severe dive profile in this study) was $1.80 \%$ in a prior analysis (Gerth and Vann 1997).

The presence of venous gas bubbles was not directly measured, but the dive profiles used were similar to other dive studies in which venous bubbles were detected by Doppler. In our ocean SCUBA dive, the average time and maximum depth was $48 \mathrm{~min}$ and $30 \mathrm{~m} / 98 \mathrm{fsw}$, while the dry chamber divers were pressurized for $60 \mathrm{~min}-18.3 \mathrm{~m} /$ $60 \mathrm{fsw}$. In a study of recreational SCUBA divers by Dunford in 2002, venous gas emboli bubbles were detected in 61/67 divers following a single 39- to 45 -min ocean dive to median max depth $26 \mathrm{~m} / 85 \mathrm{fsw}$ in the absence of decompression sickness (Dunford et al. 2002). Carturan reported in 2002 that $31 / 46$ healthy volunteer divers had venous bubbles after a 25 -min dive to $35 \mathrm{~m} / 115 \mathrm{fsw}$ (Carturan et al. 2002). In other studies, venous bubbles were detected in $4 / 8$ subjects after $30 \mathrm{~min}$ of ocean diving to $39 \mathrm{~m}$ (Dujic et al. 2005a), in 11/12 divers after a 30-min ocean dive to $30 \mathrm{~m}$ (Dujic et al. 2005c), in 10/10 hyperbaric chamber divers following $75 \mathrm{~min}$ exposure to $18 \mathrm{~m}$ (Valic et al. 2004), and 4/11 chamber divers following a 60 minute exposure to $60 \mathrm{fsw}$ (Spencer 1976). These studies provide neither the minute-to-minute time-depth profiles to permit calculation of a dive severity index, nor factors such as temperature and exercise which are known to affect bubble formation, and which vary between studies (Jankowski et al. 1997; Wisloff and Brubakk 2001). Further, identical dive profiles may result in different bubble grades even with similar age and body size, as exemplified to two studies to $280 \mathrm{kPa}(29 \mathrm{~m} / 94 \mathrm{fsw})$ for $80 \mathrm{~min}$. Even with similar diver 
demographics the integrated bubble load differed significantly (0.98 bubble $\mathrm{cm}^{-2}$ (Dujic et al. 2004) vs. 0.0125 bubbles $\mathrm{cm}^{-2}$ (Brubakk et al. 2005)). Thus, we cannot say with complete certainty that venous bubbles were present in our subjects; only that we believe VGE formation was highly likely.

\section{$V_{\mathrm{A}} / Q$}

We initially hypothesized a worsening of $V / Q$ mismatch, with subsequent resolution due to the transient occlusive effects of VGE on pulmonary circulation. Our hypothesis was supported by preliminary work from Einar Thorsen following a 300-m saturation dive (in which venous bubbles were detected) with associated increased slope of intrabreath $V_{\mathrm{A}} / Q$ or greater mismatch of ventilation-perfusion in the lung (Cotes 1993). What we found instead was a decreased range of $V_{\mathrm{A}} / Q 24 \mathrm{~h}$ following the SCUBA dive. The lack of change in $V_{\mathrm{A}} / Q$ following the chamber dive or $1 \mathrm{~h}$ following the SCUBA dive was interpreted to mean that VGE (if present) do not worsen ventilation-perfusion mismatch as they were expected to be formed after the chamber dive and to be cleared by $24 \mathrm{~h}$. Other explanations include the possibility that VGE did not form at these dive severities (although prior studies suggest some VGE were likely, see above), that VGE were already cleared by the time measurements were formed (although many studies have detected venous bubbles at $80 \mathrm{~min}$ post dive (Dujic et al. 1993; Valic et al. 2004)), that our population size was too small to detect a significant change, and that VGE within the lung do not form diffusely, or they lodge within distal capillaries and thus are not detected by our methods. Any contribution from exercise in the diving group would have been expected to increase $V_{\mathrm{A}} / Q$ inequality (Wagner 1992).

We employed the single-breath $V_{\mathrm{A}} / Q(\mathrm{iV} / Q)$ technique as a previously validated method for determining such changes in ventilation-perfusion mismatch (West et al. 1957; Guy et al. 1976; Prisk et al. 2003). In this model, the degree of $V_{\mathrm{A}} / Q$ inequality in the entire lung is gaged from the slope of an intra-breath $V / Q$ versus volume expired curve during phase III of expiration (prior to the onset of airway closure). In a dog model, the slope of first half of phase III correlated with independent measures of $V_{\mathrm{A}} / Q$ range including multiple inert gas elimination technique (MIGET) (Prisk et al. 2003).

Circulatory changes after diving include an increase in pulmonary artery pressure and decreased cardiac output (Dujić et al. 2006). Thus it may be that there is a more homogenous distribution of pulmonary blood flow in the lung which improve $V_{\mathrm{A}} / Q$ mismatch 24 -h after ocean-diving. This would be consistent with a concurrent decrease in alveolar dead space 24 -h post-dive, which has been shown to reflect more homogeneous distribution of pulmonary perfusion (Severinghaus and Stupfel1957). Why this was not seen at $1 \mathrm{~h}$ is unclear but again may be due to our small sample size. Also, submersion can causes central pooling of blood due to peripheral vasoconstriction (Dujic et al. 2005a), which may improve pulmonary circulation altering VGE dispersion and resolution.

\section{Spirometry}

There was a significant increase in FVC at the 1-h postSCUBA time point with a trend towards normalization by 24-h, and contrarily, a reduction in FVC 1-h after a nonexertion dry hyperbaric exposure $(p<0.05)$. The later result is in concordance with the common idea that SCUBA diving causes a decrease in FVC; however, review of the literature reveals mixed results from multiple studies, most with small subject numbers. Skogstad reported a 1996 study of 17 divers performing an ocean bounce dive to $10 \mathrm{~m} / 53 \mathrm{~min}$ or $50 \mathrm{~m} / 38 \mathrm{~min}$ in which $\mathrm{FVC}$ was reduced 5.8 and $1.8 \%$ (Skogstad et al. 1996). In that study the change in FVC was inversely proportional to dive severity, similar to our observed decreased $V_{\mathrm{A}} / Q$ inhomogeneity following more severe dives. Alternately, in a 2005 study by Dujic of eight volunteer recreational divers to $39 \mathrm{~m}$ for $30 \mathrm{~min}$, there were no changes in spirometric testing 90 minutes after a dive (no data at 24 h) (Dujic et al. 2005a). Tetzlaff observed a reduction in FVC only after a cold-dive exposure to $50 \mathrm{~m}$, but not following an ambient water temperature exposure to $50 \mathrm{~m}$, and not following cold water exposure to $10 \mathrm{~m}$ (Tetzlaff et al. 2001). In a relatively large recreational dive study comparing wet versus dry hyperbaric chamber exposures to $0.6 \mathrm{MPa}$ (61 $\mathrm{m}$ water) for 15 min with ( $n=19,22)$, no significant changes were observed in spirometric values or $D_{\mathrm{L}} \mathrm{CO}$ at 3 and $24 \mathrm{~h}$ postdive time points (Tetzlaff et al. 1999). Thus, wet exposure and immersion alone may not be responsible for changes in pulmonary function, but bronchoconstriction due to cold was thought to be likely. Thus, with relatively comfortable summer water temperatures encountered in the oceans off San Diego, a cold water effect is unlikely in this study.

\section{$D_{\mathrm{L}} \mathrm{CO}$}

The $D_{\mathrm{L}} \mathrm{CO}$ protocol was added to our study after the openocean dive data had been collected in an effort to further our hypothesis that changes in $V_{\mathrm{A}} / Q$ could be correlated with asymptomatic VGE, as previous studies had shown decreased diffusing capacity of the lung following both dry and wet dives in which VGE were detected. Following a dry-dive study to $45 \mathrm{~m}$ for $25 \mathrm{~min}, D_{\mathrm{L}} \mathrm{CO}$ was reduced significantly in proportion to maximal bubble grade (Dujic et al. 1993). Thorsen reported a significant reduction in 
TlCO following a dry dive $(n=12)$, to $0.49 \mathrm{MPa}(127 \mathrm{ft}$ sea water) for $40 \mathrm{~min}$, notable particularly in divers found to have Doppler-confirmed VGE (Thorsen et al. 1995). Finally, Skogstad reported a reduction of TlCO by 11.3 and $12.8 \%$ following ocean bounce dive to $10 \mathrm{~m} / 53 \mathrm{~min}$ or $50 \mathrm{~m} / 38 \mathrm{~min}$ in which no VGE monitoring was performed (Skogstad et al. 1996). The lack of change in $D_{\mathrm{L}} \mathrm{CO}$ following the chamber dive suggests that VGE were either not present or not sufficiently large or numerous to result in changes in $D_{\mathrm{L}} \mathrm{CO}$, despite a dive severity index of 0.94 .

\section{Hyperbaric control}

There were no observable differences in $\mathrm{iV} / Q$, alveolar deadspace, FVC, or $\mathrm{D}_{\mathrm{L}} \mathrm{CO}$ in the group of hyperbaric dive subjects who completed a second hyperbaric "bounce dive" to $60 \mathrm{ft}$ for $5 \mathrm{~min}$, when compared to their initial tests following the hyperbaric dive to $60 \mathrm{ft}$ for $60 \mathrm{~min}$. The repeat "bounce dive" was expected to be free of VGE production and thus eliminate the possibility of confounding pulmonary factors inherent to the compression-decompression process alone. Our results indicate that the effects of compression/decompression per se did not significantly influence our results.

\section{Limitations}

The major limitation to this study design was the assumption that VGE were present, in the absence of Doppler confirmation. Our dive time-depth profiles were similar to previously published studies demonstrating VGE; however, because of the inconsistency of bubble formation within a given profile and the multifactorial nature of VGE formation (including cold and exertion), it is possible that VGE were not present in our recreational ocean dive subjects or dry hyperbaric chamber subjects.

A second limitation relates to the varied exercise and dive profiles performed in the SCUBA wing of this study. Exercise during decompression has been shown to reduce bubble formation (Jankowski et al. 1997), and endurance exercise or even a single episode of exercise the day prior to diving (in a rat study) can reduce bubble formation and increase survival rate after a chamber dive to $700 \mathrm{kpa}$ (71 msw) for $45 \mathrm{~min}$ (Wisloff and Brubakk 2001). Multiple human studies have also demonstrated reduction in bubble formation associated with exercise performed $20 \mathrm{~h}$ prior to diving (Dujic et al. 2004), $2 \mathrm{~h}$ prior to diving (Blatteau, Boussuges et al. 2007), during decompression (Jankowski et al. 1997), during a 3-min decompression stop (Dujic et al. 2005b), and immediately following a dive (Dujic et al. 2006).

Other limitations relate to the small subject number (although similar to most other studies of this type with $n=11$ and $n=12$ ); however, a previous study showed that the reproducibility of the measurement was $\sim 3 \%$ in upright subjects (Prisk et al. 1995). The selection of two different study groups limits a true comparison of pulmonary function test values between the two dive exposures (open ocean or air chamber dive), and the difference in body positioning during hyperbaric exposures (prone in the SCUBA group vs. seated upright in the chamber group), which could have potentially altered patterns of blood flow and VGE clearance within the lung.

\section{Conclusion}

Contrary to our hypothesis, the distribution of $V_{\mathrm{A}} / Q$ mismatch in the lung was decreased following recreational ocean diving, and was unchanged following an equivalent air chamber dive, arguing against an impact of VGE on $V_{\mathrm{A}} / Q$ following a no-decompression stop recreational dive. While limited without Doppler confirmation of VGE, this study suggests that there are no major effects on ventilation-perfusion matching following dives of modest severity that fall within the no-decompression-stop limits.

Acknowledgments Special thanks to our volunteers and to the UCSD Hillcrest Hyperbaric Chamber staff, in particular to Jay Duchnick who led efforts to coordinate late-night chamber use and technician support. This research was funded in part by the NIH Short-Term Research Training Grant for medical students. This work was also supported by NASA contract NAS9-98124 and NASA cooperative agreement NCC9-168.

Conflict of interest statement There were no conflicts of interest for any authors in our study.

Open Access This article is distributed under the terms of the Creative Commons Attribution Noncommercial License which permits any noncommercial use, distribution, and reproduction in any medium, provided the original author(s) and source are credited.

\section{References}

Blatteau JE, Boussuges A et al (2007) Haemodynamic changes induced by submaximal exercise before a dive and its consequences on bubble formation. Br J Sports Med 41(6):375-379

Brubakk A, Duplancic D, Valic Z, Palada I, Obad A, Bakovic D, Wisloff U, Dujic Z (2005) A single air dive reduces arterial endothelial function in man. J Physiol 566(3):901-906

Carturan D, Boussuges A, Vanuxem P, Bar-Hen A, Burnet H, Gardette B (2002) Ascent rate, age, maximal oxygen uptake, adiposity, and circulating venous bubbles after diving. J Appl Physiol 93:1349-1356

Cotes J (1993) Assessment and application in medicine. Blackwell, Oxford

Direction of Commander, N. S. S. C. (2008) Navy diving manual. SS521-AG-PRO-010. U. S. Navy, Washington, DC

Dujic Z, Eterovic D, Denoble P, Krstacic G, Tocilj J, Goxovic S (1993) Effect of a single air dive on pulmonary diffusing capacity in professional divers. J Appl Physiol 74:55-61 
Dujic Z, Duplancic D, Marinovic-Terzic I, Bakovic D, Ivancev V, Valic Z, Eterovic D, Petri N, Wisloff U, Brubakk A (2004) Aerobic exercise before diving reduces venous gas bubble formation in humans. J Physiol 555(3):637-642

Dujic Z, Bakovic D, Marinovic-Terzic I, Eterovic D (2005a) Acute effects of a single open sea air dive and post-dive posture on cardiac output and pulmonary gas exchange in recreational divers. Br J Sports Med 39(e24)

Dujic Z, Palada I et al (2005b) Exercise during a 3-min decompression stop reduces postdive venous gas bubbles. Med Sci Sports Exerc 37(8):1319-1323

Dujic Z, Palada I, Obad A, Duplancic D, Brubacck A, Valic Z (2005c) Exercise-induced intrapulmonary shunting of venous gas emboli does not occur after open-sea diving. J Appl Physiol 99:944-949

Dujic Z, Obad A et al (2006) Venous bubble count declines during strenuous exercise after an open sea dive to $30 \mathrm{~m}$. Aviat Space Environ Med 77(6):592-596

Dujić Z, Obad A, Palada I, Valic Z, Brubakk A (2006) A single open sea air dive increases pulmonary artery pressure and reduces right ventricular function in professional divers. Eur J Appl Physiol 97(4):478-485

Dunford R, Vann R, Gerth W, Pieper C, Huggins K, Wacholtz C, Bennett P (2002) The incidence of venous gas emboli in recreational diving. Undersea Hyperb Med 29(4):247-259

Gerth WA, Vann RD (1997) Probabilistic gas and bubble dynamics models of decompression sickness occurrence in air and nitrogenoxygen diving. Undersea Hyperb Med 24(4):275-292

Guy H, Gaines R, Hill P, Wagner P, West J (1976) Computerized, non-invasive tests of lung function. Am Rev Respiratory Dis 113:737-744

Jankowski L, Nishi R, Eaton D, Griffin A (1997) Exercise during decompressure reduces the amount of venous gas emboli. Undersea Hyperb Med 23(2):71-75

Marx J (2006) Rosen's emergency medicine: concepts and clinical practice

Prisk G, Elliott A, Guy H, Kosonen J, West J (1995) Pulmonary gas exchange and its determinants during sustained microgravity on Spacelabs SLS-1 and SLS-2. J Appl Physiol 79(4):1290-1298

Prisk G, Guy H, West J, Reed J (2003) Validation of measurements of ventilation-perfusion ration inequality in the lung from expired gas. J Appl Physiol 94(1186-92):1186-1192
Sackner M, Greeneltch D, Heiman M, Epstein S, Atkins N (1975) Diffusion capacity, membrane diffusing capacity, capillary blood volume, pulmonary tissue volume and cardiac output measured by a rebreathing technique. Am Rev Respiratory Dis 111:157165

Severinghaus J, Stupfel M (1957) Alveolar dead space as an index of distribution of blood flow in pulmonary capillaries. J Appl Physiol 10:335-348

Short D, Flahan M (1989) Reconstructing the Navy tables. AAUS DC workshop. U.S.C. Sea Grant Program, Los Angeles

Skogstad M, Thorsen E, Haldorsen T, Melbostad E, Tynes T, Westrum B (1996) Divers' pulmonary function after open-sea bounce dives to 10 and $50 \mathrm{~m}$. Undersea Hyperb Med 23(2):71-76

Spencer M (1976) Decompression limits for compressed air determined by ultrasonically detected blood bubbles. J Appl Physiol 40(2):229-235

Tetzlaff K, Staschen C, Koch A, Heine L, Kampen J, Neubauer B (1999) Respiratory pattern after wet and dry chamber dives to $0.6 \mathrm{MPa}$ ambient pressure in healthy males. Respir Physiol 118(2/3):219-226

Tetzlaff K, Friege L, Koch A, Heine L, Neubauer B, Struck N, Mutzbauer T (2001) Effects of ambient cold and depth on lung function in humans after a single scuba dive. Eur J Appl Physiol $85(1 / 2): 125-129$

Thorsen E, Risberg J, Segadal K, Hope A (1995) Effects of venous gas microemboli on pulmonary gas transfer function. Undersea Hyperb Med 22(4):347-353

University of California-Scripps Institute of Oceanography (2007) Shore stations program - La Jolla, California. http://shorestation. ucsd.edu/active/index_active.html

Valic Z, Dulancic D, Bakovic D, Ivancev V, Wisløff U, Brubakk A, Dujic Z (2004) Diving-Induced venous gas emboli do not increase pulmonary artery pressure. Int J Sports Med 26:626-631

Wagner PD (1992) Ventilation-perfusion matching during exercise. Chest 101(5 Suppl):192S-198S

West J, Fowler K, Hugh-Jones P, O’Donnell T (1957) Measurement of the ventilation-perfusion ration inequality in the lung by the analysis of a single expirate. Clin Sci 16:529-547

Wisloff E, Brubakk A (2001) Aerobic endurance training reduces bubble formation and increases survival in rats exposed to hyperbaric pressure. J Physiol 537(2):607-611 